\title{
Two-photon photoluminescence induced defects on InGaN crystal and light emitting diodes
}

\begin{abstract}
Two-photon excitation techniques used in fabricating lines defects were done on a light emitting diode chip. Simultaneous detection of a quenched wide-gap semiconductor crystal has been observed using single- and two-photon photoluminescence. It was found at the quenched area, single-photon excitation gives photoluminescence read-out compared to twophoton excitation which no photoluminescence detected at the bandgap wavelength. This is due to the excitation states which the transition of electron for two-photon excitation to occur have been demolished by the annealing of the sample which involved two-photon quenching process. The dependency of excitation power with respective photoluminescence is elaborated to confirmed the single- and two-photon excitation photoluminescence methods.
\end{abstract}

Keyword: Photoluminescence; Quenching; Single-photon; Two-photon 